\title{
Engineering plasma spray films by knowledge based simulation
}

\author{
M. Foy, \\ Vitalita, Inc., 2155 South Walnut Street, Unit 8 \\ Boulder, Colorado 80302-453, USA \\ Telephone: +1-303-938-8928, Fax: same upon request \\ E-mail:mfoy@geocities.com \\ M. Marchese and G. Jacucci, \\ Università di Trento, Laboratorio di Ingegneria Informatica, \\ Dipartimento di Informatica e Studi Aziendali, Via Zeni 8, \\ Rovereto (TN), Italy \\ Telephone: +39-464-443132/43, Fax: +39-464-443141 \\ E-mail: \{marchese; gianni\}@lii.unitn.it
}

\begin{abstract}
The FABERCOAT Decision Support System (DSS) is a design tool for the production of plasma-sprayed coatings developed in a CEC project among European universities, laboratories and manufacturing industries. The FABERCOAT system utilises a genetic algorithm to find a set of spray settings which can be used to achieve one particular set of desired final (and measurable) properties of the coating. At present, these desired outcomes are based on specific industrial requirements based on total porosity demands and most frequent porosity aspect ratio. The FABERCOAT system includes a model for the plasma spray manufacturing process developed and validated within the CEC project. The model has been used to "harvest" the information gained in the extensive production and characterisation programme of the CEC project and has provided an innovative way of feeding back this information to the end-users.
\end{abstract}

Keywords

Coating manufacturing, decision support systems, expert systems, knowledge based simulation, genetic algorithms,

The original version of this chapter was revised: The copyright line was incorrect. This has been corrected. The Erratum to this chapter is available at DOI: 10.1007/978-0-387-35357-9_22 


\section{INTRODUCTION}

In the following the concepts and the implementation of a prototype Decision Support System (DSS) for the manufacturing of plasma-sprayed coatings named FABERCOAT will be presented and discussed. The name is taken directly from the name of the European research project (Fabercoat, 1995) under the BRITE/EURAM programme on the characterisation and modelling of the manufacturing process of plasma-sprayed coatings.

Briefly, the FABERCOAT System can be used to find a set of spray settings in order to achieve one particular set of desired final (and measurable) properties of a coating. The system is based on the knowledge imbedded in a model for the deposition process that provides microstructural information (such as total porosity and/or porosity aspect ratio distributions) that are needed for the evaluation of a coating's effective properties.

The aims of the FABERCOAT System are:

- to simplify the use of deposition models in order to simulate the spraying process in a fast and effective way;

- to use the information of theoretical and experimental research in order to provide computerised decision support to people who perform plasma spraying manufacturing.

The final aim is to allow operators to specify what coating characteristics are desirable (porosity/temperature profile/residual stress profile), and ask the DSS to give suggestions on how to configure the spraying operation.

Section 2 focuses on the definition of the concepts and the user requirements for the FABERCOAT System as they emerged in the course of the project. Section 3 is centred on the description of the implemented prototype and on its application in specific cases.

\section{FABERCOAT SYSTEM CONCEPTS}

The FABERCOAT System is centred around a model for the plasma spray manufacturing process developed and validated within the FABERCOAT project (Fabercoat, 1995). A deposition model has been developed and used to "harvest" the information gained in the extensive production and characterisation programme of the project and has provided an innovative way of feeding back this information to the end-users (engineers and technicians at the production laboratories).

Specifically the FABERCOAT System consists of a deposition model for the plasma spray manufacturing process, together with two components: (1) a graphic user interface (GUI) to interface/communicate between operators and the model; (2) an expert system component that co-ordinates the execution of the model.

The expert system component is a so called a "front-end system" (O'Keefe, 1986). In fact, in our system, this component acts as a "front-end" for defining a numerical simulation which is subsequently run on its own. 


\section{FABERCOAT \\ System Concept}

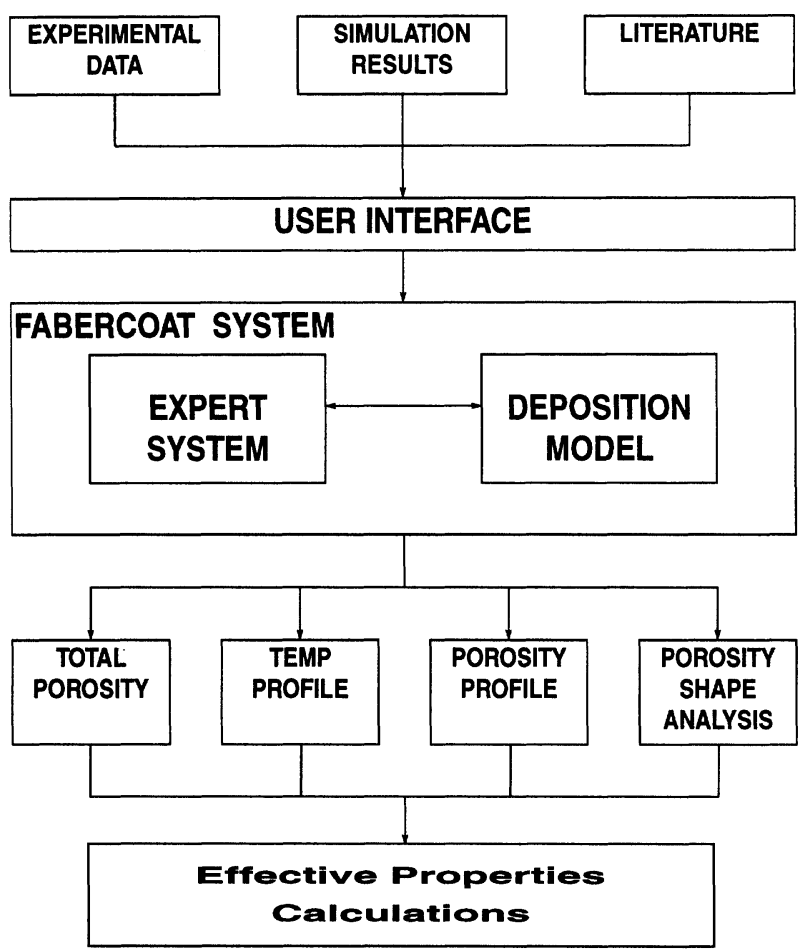

Figure 1: FABERCOAT System Concept.

The solution space of a great number of numerical simulations are automatically analysed and searched for the desired properties. The expert system component is used:

- to combine project information and knowledge into one tool;

- to allow a user to perform intelligent and automatic searches of the spray parameter space;

- to assist final users in performing reliable correlations between initial spray parameters and final coating properties.

The overall scheme of the FABERCOAT System is summarised in Figure 1. 


\subsection{Basis for the Development of the FABERCOAT System}

Before describing the FABERCOAT System functionality, we recall here the main results of previous investigation (Fabercoat, 1995) that have been relevant in the definition of the FABERCOAT System.

In regard to the experimental investigations, the FABERCOAT System relied on the following results:

i - collection of experimental data on the plasma spray manufacturing processes at the three different length scales of the process: a) data on the single droplet of ceramic particle (in flight and after impact on the substrate), b) data on the mechanism for the assembly of droplets, c) information on the macroscopic properties of the final coatings;

ii development and use of new techniques to measure plasma-particle interactions to provide the necessary data to the model of the deposition process;

iii- development of image analysis tools to characterise coating microstructures (porosity, crack network fractal dimension);

iv X-ray analysis to measure phase composition and residual stresses.

In regard to the modelling and simulation investigations, the following results have been used in the Decision Support System (DSS):

1 - a deposition model (2-d) for the plasma spray manufacture of ceramic coatings;

2. theoretical and numerical models for the description of the mechanical and the thermal material behaviour of ceramic layers;

3. a residual stress model to estimate strain and stresses developed in the coating during fabrication.

The deposition model has been identified as the central component in order to fulfil the DSS goals, namely to try to correlate process parameters and final coating characteristics. The main capabilities of the deposition model (Cirolini et al, 1991 and 1995) consist of predicting a number of final coating microstructural characteristics (such as total porosity, porosity profile and shape distributions, and temperature distributions) that can be used to determine, in a second stage, final coating mechanical and thermal properties (among others, elasticity modulus and thermal conductivity). In the following we review briefly the input data needed for the deposition model; in turn they represent the necessary input data for the FABERCOAT System:

(1) correlation between torch settings and plasma and particle properties, i.e.:

- data on plasma gas (mainly temperature) as a function of torch settings and gun distance;

- data on in-flight particle characteristics such as particle radius, velocity and temperature distributions as a function of torch design, settings and distance.

Such detailed data are complex to measure, but have been obtained in a complete form for one specific torch in the course of the Fabercoat project (Fabercoat, 1995) as well as in other research work (Fauchais, 1989). It is useful to underline that 
significant effort has been applied in the last 10 years in the plasma-spray community to model the plasma gas and the transport of particles in it (Vardelle, 1986). By now several models have been developed and validated for some plasma torches, and are commercially available. They could be easily integrated in the FABERCOAT System to extend the needed correlation between torch settings and plasma and particle properties for other apparatus. For this prototype version of the deposition model we have limited our simulation to consider only the acquired experimental data for one torch.

(2) Correlation between in-flight and after-impact particle properties, i.e.: data on the behaviour of particles after impact as a function of initial conditions.

Theoretical and numerical models of the impact process have been developed in the past 5 years in order to obtain information on the particle flattening degree as a function of a particle's initial conditions and material properties (Trapaga et al, 1991 and 1992, Fukai, et al, 1993, Bertagnolli et al, 1995). The available information has been integrated in the present version of the FABERCOAT System.

\subsection{FABERCOAT System functionality}

The FABERCOAT System provides multiple levels of functionality so different types of deposition model simulations can be easily carried out, and results can be examined as to their quality.

\section{Multiple simulation executions}

The first necessary functionality allows the user to execute multiple simulations automatically. Users often would like to run multiple simulations (possibly varying one spray parameter to see how that effects one or more of the overall characteristics of the final coating), but this task can often be laborious, as the user has to continuously monitor the results, change input files, and then reiterate simulations. The FABERCOAT System alleviates this difficulty by allowing the user to quickly specify a multiple simulation cycle, and have this cycle automatically performed without user intervention.

\section{Expert system component for intelligent search}

Furthermore, beyond this simple iterative simulation functionality, this system integrates an expert system component, which intelligently performs more complicated simulation cycles. This expert system component intelligently directs multiple simulation cycles, specifically, it performs intelligent search. Explicitly, the component executes a search within the space of spray parameters, with the goal to locate a particular set of coating characteristics. For example, a user is able to specify a certain set of desired outputs (such as a desired total porosity of 10\%), and then direct the system to find a set of spray parameters (within certain ranges) that most closely matches the set of desired outputs. The system then responds as an expert would, by giving advice about how to set the spray conditions (gun 
settings, substrate conditions, etc.) in order to achieve the desired properties in the final coating.

\section{Simulation history archiving}

Within these iterative simulation cycles (either directly or through search), the system is also be able to archive all results from all simulations executed. For example, when performing a search for a set of spray parameters, the system maintains a "history file" containing a list of all the simulations which have been performed. This is useful for at least three reasons. First, this permits the user to go back and browse the simulation history, so that trends and correlations between model inputs and model outputs (i.e., between spray parameters and final coating characteristics) can be discovered. Second, by maintaining a simulation history, if the search were to terminate before finishing, all results would not be lost, and it may be possible to start the search off from where it left off. This is an important issue to some users because the deposition model can take considerable computation time, and therefore, every simulation is valued and they may want to look at the results from all simulations that have been performed. Lastly, a large set of simulations results can be useful to model developers because it can help them detect bugs in the simulation model computer code, so that these bugs can be fixed.

\section{$N$-best members produced during search}

In addition to archiving a complete history, when the system is performing a search, a set of n-best solutions is maintained, and available for review at the end of the search. That is, the n-best set of spray parameters which give results most closely matching those specified as most desirable by the user are recorded. Therefore, at the end of a search, not only is the best set of spray parameters presented, but the n-best (e.g., 20-best) that have been encountered during the entire search. This is useful so the user can see a number of spray settings that will match her needs, rather than getting only one "answer", and also allow her to see how much "spread" (i.e., separation or distance) there is between good sets of spray settings.

\section{Graphical user interface}

All of these functionalities are contained within a graphical user interface (GUI) that easily allows the user to execute these tasks. In particular, the user is able to specify parameters and preferences within the GUI (using type-in boxes, etc.), and then carry out actions with the use of buttons.

Overall, these functionalities should provide users useful information and decision support by permitting them to structure simulations and searches to assist in the understanding of the deposition model. Therefore users should gain a better understanding of the mechanics of the deposition model, and plasma spray processes, because trends in the model, and correlations between inputs and outputs, will be much easier to observe. 


\subsection{The deposition model parameters}

The deposition program has a long list of input parameters that can be divided in three main groups: "operator" - "physical" - "computational".

The "operator" group includes, for example, the gun distance during the spray, the ceramic powder granulometry, and the final thickness of the coating. These parameters are supposed to be directly established by users and users should specify them in standard "engineering" units.

The "physical" group includes, for example, the particle's velocity and temperature at splat time. These values describe the current state of the physical process that is being simulated; they refer to quantities not directly established in real life but that depend on those in the first category by known relations, either analytical, experimental or produced by other simulations.

The "computational" group includes parameters related to the inner workings of the deposition program like grid sizes, random generator seeds and so on. These parameters affect the "precision" and the run time of the program and may depend on those in the "operator" and "physical" classes. For example, the grid size depends on the size of the particles and on the final thickness of the coating (for more information see Cirolini et al, 1991 and 1995).

Originally the second class (i.e., the "physical" group) was the most numerous one because the deposition program was correlating basic physical quantities. The availability of experimental measurements (in-flight velocity and temperature as a function of torch settings) and the results of other simulations (particle splashing and curling for different temperature and velocity combinations) allows for filling the gap between real life parameters and physical parameters thus increasing the size of the "operator" class and reducing the "physical" one.

\subsection{Selection of the searchable spray parameters}

A subset of the above deposition model input parameters were chosen for inclusion in the set of spray parameters which may be "searched over" by the expert system component. This selection focused on the most important operator-controlled spray parameters which have the most important effect on the final coating characteristics.

Functionality has been included which allows the user to choose which of these parameters should be search over (including what range they should be searched) and which should not be searched, but left at particular constant values. This allows the user the flexibility to decide what parameters are available to be changed in the spraying process, and which ones should remain fixed due to some limitation of the spraying process. This is an important point because the final goal is to make the program usable by operators, not only by physicists or programmers.

The searchable spray parameters, with their corresponding values or ranges, are:

\begin{tabular}{|c|c|c|}
\hline TA & $0.075-0.15 \mathrm{~m}$ & distance between gun and substrate \\
\hline GUNVELOC & $0.02-0.25 \mathrm{~m} / \mathrm{sec}$ & velocity of the gun \\
\hline PARTDIAM & $20-45$ microns & diameter of particles \\
\hline
\end{tabular}




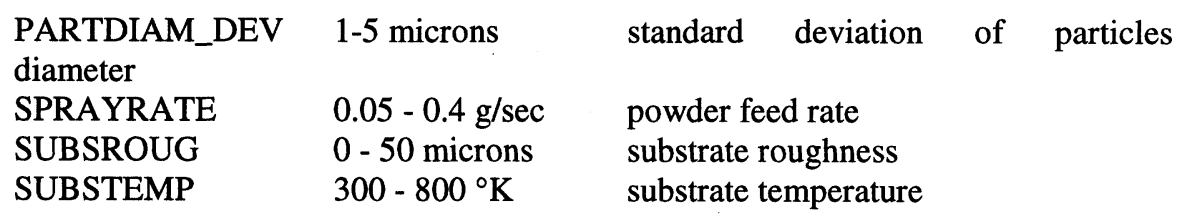

\subsection{Selection of the objective function}

The objective function is the physical property of the coating the operator wants to control, i.e. the quantity in the output of the deposition program for which the operator wants to choose a desired value and have the Expert System component search for the unknown parameters.

The outputs of the deposition program at present include: a simulated micrograph of a section of the coating, the intermediate and final temperature profile (through the coating thickness), the intermediate and final porosity profile, the final pore aspect ratio distribution and the final unmelted particle percentage.

The simulated section of the coating can be compared to a microscope image of a section of the coating, and allows one to see pores and individual splats. It gives an overall view of the coating, but its information is hard to translate into a quantitative one, and it is better used as a visual check to indicate if something wrong happened in the simulation.

The through-thickness temperature profile is available at different times during the deposition, the last one being especially important for the successive development of residual stresses during cooling. Temperature is usually fairly constant in the substrate, with the exception of a sharp change at interface and quite a big gradient in the coating. The information contained in the temperature profile could be reasonably summarised in one number by taking the gradient in the coating, or measuring the temperature step at the interface.

The porosity profile is probably the most interesting result. Porosity influences the mechanical properties of the coating and can be correlated with measurements done on real coatings. As with the temperature profile there is the problem of compressing its information to just one number. A reasonable first approximation is to compute from the porosity profile the total mean porosity of the coating section.

Another output parameter is the distribution of the aspect ratio of the pores: its importance relies on its strong influence on the mechanical and thermal effective properties of the coating (Rickerby et al, 1987 and McPherson, 1989). The typical feature of these types of distributions are the abundance of thin, elongated pores (like cracks) with an aspect ratio less than 0.3 in the simulated coating. These cracks are found to have the greatest effect on the mechanical and thermal effective properties of the ceramic.

The last output parameter is the unmelted particle percentage. Apart from correlating strongly with the final mean porosity present in the coating, the amount of unmelted material needs to be controlled during fabrication to assure the overall quality of the coating. 
In the first development phase of the FABERCOAT System, it was decided to use the total mean porosity as the objective function for its smooth behaviour. The other outputs have been provided in this phase as a check on the correctness of the results from the prototype system.

\section{FABERCOAT SYSTEM PROTOTYPE}

A prototype FABERCOAT System that utilises the concepts presented in the last sections has been developed and is under use in the plasma-spray laboratory of project's partners.

\subsection{Search method}

As described in a previous section presenting the FABERCOAT System functionalities, the system has the capability to perform intelligent searches of the spray parameter space (i.e., a search through all possible sets of spray parameters). This is directed by an expert system component. Due to the requirements of this component, a specialised search method was selected to play a part in the expert system component.

In this particular domain of ceramic thermal coating processes, where knowledge of the domain is hard to codify (i.e., 'rules of thumb' are vague and difficult to construct), the selection of a search method for the expert system component is a good choice. This is due to the fact that, in general, search methods do not rely on 'rules of thumb', rather, rules are not required and an intelligent search algorithm can actually facilitate the user in identifying 'rules of thumb'.

The selection of the actual search method was made among the following possible methods: hill-climbing, simulated annealing, and genetic algorithms. In the end, genetic algorithms (GAs) were selected as the most desirable method because:

(a) they can perform unbiased search,

(b) they make no assumptions about the search space (i.e., the search space does not have to be smooth or regular),

(c) they carry out a more effective search of an irregular, multi-dimensional space because they search from a population of points rather than a single point,

(d) their search is not random, but intelligent (they utilise operators which are patterned after natural genetics), and

(e) they have been shown effective at finding optimal or near-optimal solutions to dynamic real-world problems (Holland, 1975 and Goldberg, 1989).

For a complete description of GAs, how they function, etc., refer to Holland (1975) and Goldberg (1989) 


\subsection{Linking the deposition model and the genetic algorithm (GA)}

To allow the GA to search the space of the deposition model spray parameters, the deposition model is linked to the GA, and the GA uses the deposition model as the evaluation function.

Therefore, whenever the GA wants to evaluate the "worth/fitness" of a set of spray parameters, the deposition model is called, and the final outcome is returned to the GA so that a fitness can be computed. This new hybridised-system component is called the "deposition model-GA" or the DEP-GA. Figure 2 illustrates how the deposition model (DEP model) and GA are linked to form the DEP-GA component.

For the DEP-GA to find a near-optimal spray parameter set for given desired final coating characteristics, it goes through three primary steps.

First, the DEP model and the GA are initialised. The initialisation of the GA involves establishing an initial-random population of spray parameter sets which includes only the spray parameters that are being searched (these are called population members, and are represented within the GA as bit strings).

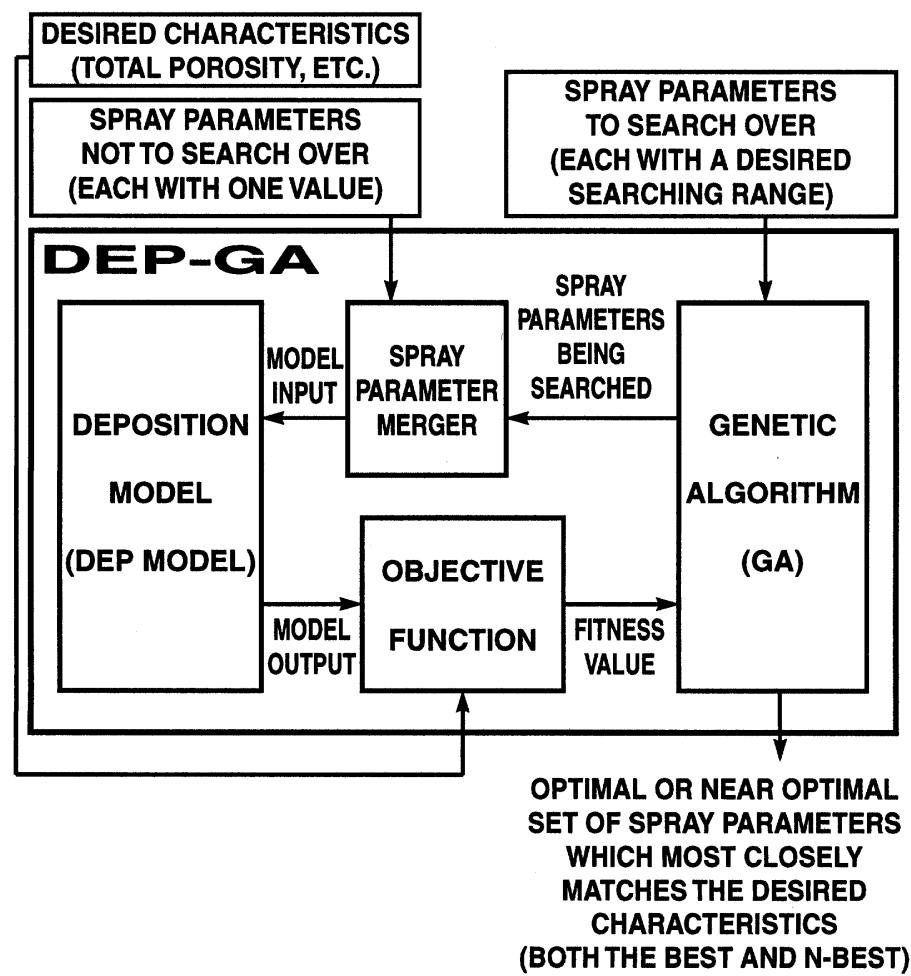

Figure 2: Structure of the DEP-GA. 
The second main step in the DEP-GA is the fitness computation. This involves taking each GA population member (one set of searchable spray parameters) filling in the spray parameters which are not being searched with base values, and executing a DEP model simulation with this full set of spray parameters. The outputs that come from this execution are then passed through the objective function (which can be structured by the user - see Section 2.5), matched against the desired characteristics, and finally a fitness value is produced which is then returned to the GA. This fitness evaluation step is executed many times because new population members are generated by the GA at the end of each generation cycle.

The last main step is the evolution of the GA population. This involves manipulations on the bit strings (i.e., operations on the population members). The three manipulations, or operators, used in the DEP-GA are reproduction, crossover, and mutation (descriptions of these operators can be found in Goldberg, 1989). GA evolution is usually continued until the GA has converged on a optimal or nearoptimal set of spray parameters which best matches the desired characteristics (total porosity, etc.) specified by the user. Lastly, the best and n-best set of spray parameters are produced for inspection by the user.

\subsection{DEP-GA performance}

Multiple sets of DEP-GA test runs have been done within the FABERCOAT System. As a starting point for evaluating the effectiveness of the DEP-GA, we chose an objective function which only included the total porosity (even though we could have also used the final coating temperature or the unmelted particle percentage as well - see Section 2.5 for more information). Additionally, we limited the spray parameters to search over to three: GUNDISTA, PARTDIAM, and SPRAYRATE (as described in Section 2.4). The below sub-sections describe the results obtained within this scenario.

\section{GA convergence on an optimal set of spray parameters}

The DEP-GA has performed similarly to most GAs, in that during a run, the average fitness of the populations, over time, has increase. That is, the members in the later populations have converged on maximum members in the space (i.e., on optimal sets of spray parameters). Figure 3 shows that the DEP-GA does in fact produce this typical performance. Specifically, this figure illustrates the evolution of fitness values coming from a DEP-GA run where the user has specified to find a set of spray parameters which will give $10 \%$ overall porosity. Note that as generations proceed, the GA weeds out members which do not produce the desired results (i.e., members that have low fitness) and focuses on members that produce coatings near the desired $10 \%$ porosity (i.e., members that have high fitness). Also note that by the fifth generation, the GA has probably already converged on the optimal spray parameters because the maximum fitness does not increase after the fifth generation, and because the average fitness does not make any significant 
upward trends after the tenth generation (in fact our full space investigations have shown that the maximum member in the fifth generation was the best member in the entire space).

This behaviour supports the hypothesis that the GA run need not be run for 60 generations (as shown in Figure 3), but could be terminated between the fifth and tenth generation and still obtain very good results. Preliminary statistical runs have confirmed this hypothesis.

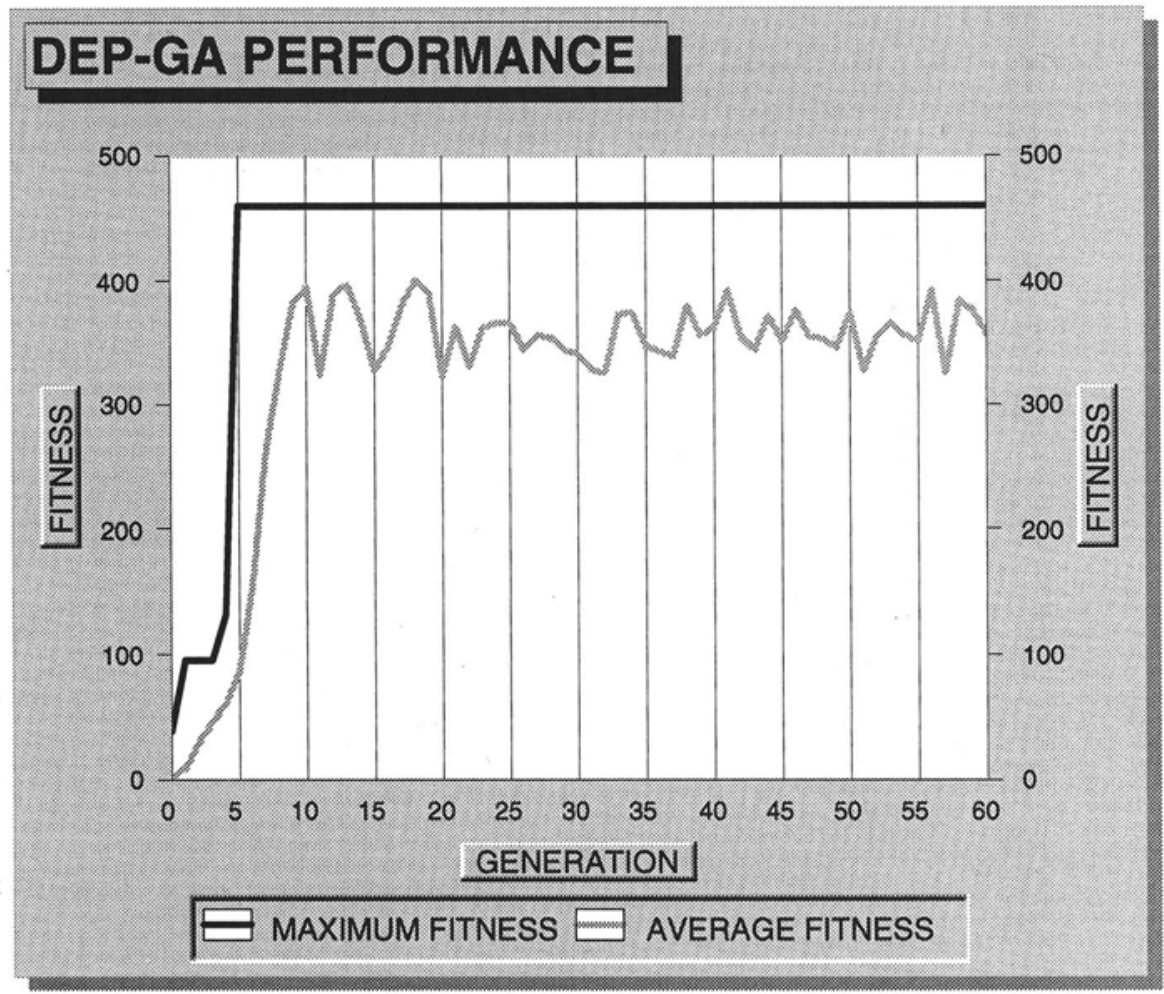

Figure 3: DEP-GA Performance.

\section{Locating $n$-best sets of spray parameters}

The above discussed performance is a positive indictor of how well the GA has been able to find a good set of spray parameters that match the user's specified needs (and how well we expect it to work in the future under different objective functions and search parameter scenarios).

For more specific results a number of runs have been performed on the chosen subset of the parameter's space, namely the subset referring to three spray 
parameters: GUNDISTA, PARTDIAM and SPRAYRATE. For this case, a complete run of the deposition model has been made in order to visualise the whole space (i.e., all possible combinations of these three spray settings within reasonable ranges have been run, and the results have been combined). Figure 4 displays the total porosity results, as produced by the deposition model, as a function of SPRAYRATE (different sections, range $0.04-0.4 \mathrm{~g} / \mathrm{sec}$ ), GUNDISTA (x-axis, range $0.075-0.15 \mathrm{~m}$ ) and PARTDIAM (y-axis, range $20-45$ microns). Contour lines refers to different porosity levels ranging from 5 to $19 \%$; each shade indicates a $2 \%$ interval of porosity. Some important features of this space can be underlined: (a) porosity tends to increase with increasing gun distance, and tends to decrease with decreasing spray rate, (b) porosity distribution is quite similar for most spray rate values, and (c) there exists different disconnected regions where total porosity values are equal (i.e., the model predicts different disjoint sets of parameters that result in the same value of final total porosity) (note for example the tile in the middle-right of Figure 4, there is a 9-11\% porosity region in the upper-right of this tile, and also in the lower part of this tile, and these regions are separated by a region with $11-13 \%$ porosity).

The black dots in Figure 4 indicate the n-best members (in this case, 20-best members, i.e., the best, 2nd best, 3rd best, etc.) found by the DEP-GA run described above (i.e., targeting $10 \%$ total porosity, and searching over only 3 spray parameters). As can be seen, because a diverse set of n-best members has been produced, the GA has not searched just one part of the space, but has been able to search different parts of the space. Additionally, because this n-best tracking has been included, the GA can indicate more than just one set of parameters that will give the desired porosity, but a number of sets of parameters (which may be spread out over the whole space) that will give results very near to the desired porosity.

This allows the expert system component to provide a widely distributed set of near-optimal spray parameter sets, thereby giving the user the opportunity to examine many different solutions, and focus on the one that most closely matches her needs (e.g., the user may like to avoid solutions which give particular types of porosity shapes, or a particular percentage of unmelted particles).

Overall, taking into consideration the results from the above described scenario, and additionally other test runs, we have found the FABERCOAT System with the DEP-GA to perform very well. Furthermore, we expect that when a real-user utilises the DEP-GA, it will produce good sets of possible spray parameters which the operator can then use to determine the actual spray parameters to use in a real spraying process. This is provided by the searching capabilities of the GA.

\subsection{Computer aspects}

\section{Graphical user interface}

The FABERCOAT System integrates a graphical user interface (GUI) around the deposition model and genetic algorithm, thereby making the system easier to use. This GUI is implemented using a public domain tool kit called SUIT. One of the largest advantages of SUIT is that it makes the FABERCOAT System portable, 
that is, it will run on 4 different computer platforms: UNIX X-Window machines, IBM-PC DOS machines, IBM-PC MS-Windows machines, and Macintosh machines

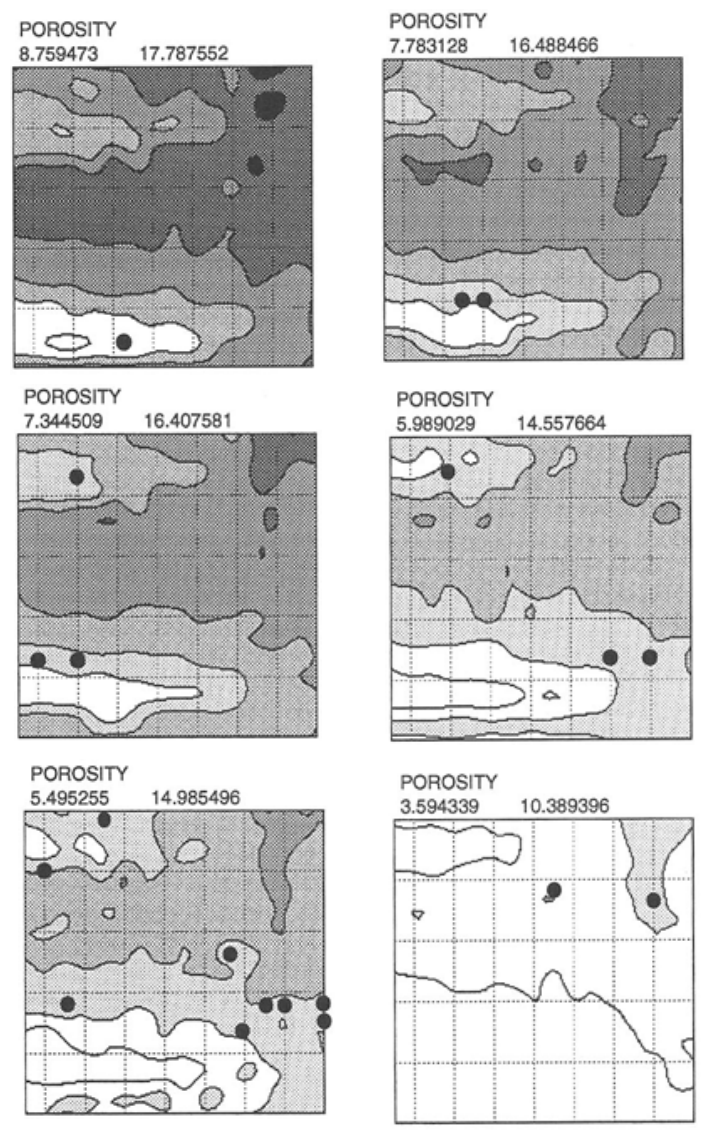

Figure 4: Sections of the three spray parameter's space indicating total porosity levels - GUNDISTA (x-axis), PARTDIAM (Y-axis), SPRAYRATE (different sections). Contour lines refer to porosity between 5 and $19 \%$, each shade indicating a $2 \%$ interval of porosity. Black dots refer to the 20 -best members found by the DEP-GA when searching for $10 \%$ porosity.

(as long as there is a deposition model compiled on the platform with the FABERCOAT System).

\section{Run time}

At present, it is most desirable to run the FABERCOAT System on a fast UNIX XWindow workstation because these machines have the greatest ability to run the 
deposition model in a reasonable amount of time (1 to 2 minutes for one simulation; about 10 hours for a full DEP-GA run), but in the future, when all machines become faster, it should even be reasonable to run the DEP-GA component on an PC type machine.

\section{CONCLUSIONS}

The prototype FABERCOAT System has proved that it has the capability to provide decision support in the area of the manufacturing of plasma sprayed coating. At the present time the prototype DSS is under use in the plasma-spray laboratories of the partners involved in the project.

The FABERCOAT System utilises a genetic algorithm (GA) to find a set of spray settings which can be used to achieve one particular set of desired final (and measurable) properties of a coating. These desired outcomes can be based on total porosity and/or final temperature of the top of the coating and/or final unmelted particle percentage requirements. The FABERCOAT System is centred around a model for the plasma spray manufacturing process developed and validated under the CEC project. The model has been used to "harvest" the information gained in the extensive production and characterisation programme of the project and has provided an innovative way of feeding back this information to the end-users (mainly technicians at the production laboratories).

\section{REFERENCES}

Bertagnolli M. , Marchese M. and Jacucci G., (1995) Thermomechanical simulation of the splashing of ceramic droplets on a rigid substrate. Journal of Plasma Spray, 4(1), 1--9.

Cirolini, S., Harding, J.H., and Jacucci, G. (1991) Computer simulation of plasma-sprayed coatings: I. Coating deposition model. Surface and Coating Technology, 48, 137--145.

Cirolini S., Marchese M., Jacucci G. and Harding J. (1995) Modelling the deposition process of thermal barrier coatings. Journal of Plasma Spray, 4(1), $10--18$.

Fabercoat Brite/Euram Project No.BREU-0418 (1995) Fabercoat: Modeling and characterization of the manufacturing process of ceramic thermal barrier coatings, Final Technical Report, European Union, Bruxelles.

Fauchais, P., Grimaud, A., Vardelle, A., and Vardelle, M. (1989) La projection par plasma: une revue. Annales de Physique, 14, pp 261--310.

Fukai, J, Zhao Z., Poulikakos D., Megaridis M. and Miyatake O. (1993) Modeling of the deformation of a liquid droplet impinging upon a flat surface. Phys. Fluids A, 5(11), 2588-2599.

Goldberg, D.E. (1989) Genetic Algorithms in Search, Optimization, and Machine Learning. Addison-Wesley, Reading, MA, USA. 
Holland, J.H. (1975) Adaptation in Natural and Artificial Systems. University of Michigan Press, Ann Arbor, MI,USA.

McPherson, R. (1989) A review of microstructure and properties of plasma sprayed ceramic coatings. Surface and Coating Technology, 39-40, 173--181.

O'Keefe , R. (1986) Simulation and Expert Systems - A Taxonomy and Some Examples, Simulation, 46(1), 10-16.

Rickerby, D.S. , Eckold, G., Scott, K.T., and Buckley-Golder, I.M., (1987) The interrelationship between internal stress, processing parameters and microstructure of physically vapor deposited and thermally sprayed coatings. Thin Solid Films, 154, 125 -- 141.

Trapaga, G., and Szekely, J. (1991) Mathematical modeling of the isothermal impingement of liquid droplets in spraying processes. Metallurgical Transactions B, 22B, 901--914.

Trapaga, G., Matthys, E.F.,Valencia, J.J., and Szekely, J.(1992) Fluid flow, heat transfer, and solidification of molten metal droplets impinging on substrates: comparison of numerical and experimental results. Metallurgical Transactions $B$, 23B, 701--718.

Vardelle, A., Vardelle, M., and Fauchais, P. (1986) Les transfers de quantitl'e de mouvement et de chaleur plasma particules solides dans un plasma d' arc en estinction. Revue Internationelle des Hautes Temperatures et Refractaires, 23, pp 69--85.

\section{BIOGRAPHIES}

Mark Foy has a Masters Degree in Computer Science from the University of Illinois at Champaign-Urbana where his work focused on artificial intelligence, specifically genetic algorithms. He has worked for the Laboratory of Information Technologies and Communications in Rovereto, Italy from 1992 to $1995 . \mathrm{He}$ is currently an independent consultant in Boulder, Colorado, USA, and has been involved in software engineering activities for various information technology organisations such as U S WEST (telecommunications), Access Graphics (distribution), and the Bavaria Ministry of Agriculture (agricultural informatics).

Maurizio Marchese graduated in Physics at the University of Trento. Currently he is a researcher in Material Science and Computer Aided Manufacturing at the University of Trento. He has been responsible of the scientific coordination of two Brite/Euram projects on the modelling and optimisation of industrial production processes. Main research interests are: - the development and application of models for the optimisation of the manufacturing processes; - the analysis and development of software tools for decision support in the design of materials manufacturing processes. He can be reached via email at the address marchese@ lii.unitn.it. 
Gianni Jacucci received a Laurea in Physics at University of Rome - Italy (1967). Since then he has been Researcher of the Italian National Research Council (19711985) Professor of Physics at the School of Engineering, University of Trento Italy (1986-1992).Professor of Informatics at the School of Engineering, University of Trento (from 1992). Visitor at CECAM (European Computing Center for Physics), Paris - France (1973-1977). Responsible of a sw group at Istituto per la Ricerca Scientifica e Tecnologica - Trento (1981-1985). Director of the Centro di Fisica degli Stati Aggregati ed Impianto Ionico in Trento of the Italian National Research Council, active in materials science, and artificial intelligence (1983-1988). Responsible of research contracts between Universita' di Trento and Consorzio CAD-CAM Bolzano - Italy (1988- 1989), towards the establishment of a Research Center for Computer Applications. Adjunct prof. of physics and supercomputing applications, University of Illinois at U-C - USA (1990-91). Director of Laboratory of Informatics Engineering, Dept. of Computer and Management Sciences - University of Trento (from 1991). Responsible of research funding in UE Framework Programs for research, development and tecgnology transfer: ESPRIT, RACE, BRITE-EURAM, CAMAR/AIR, COPERNICUS, evaluation expert for same. Member of IFIP TC5 WG 5.2, 5.3. Prof. Jacucci's current research and academic interest are in the area of introduction of new information and communication technologies and of its interaction with the enterprise organisation. 